\title{
Detection and apparent taste intensity of salt-acid mixtures in two media
}

\author{
ROSE MARIE PANGBORH AND IOA M. TRABUE
}

UNIVERSITY OF CALIFORNIA, DAVIS

Gustatory responses to mixtures of sodium chloride and citric acid were measured in two media of dispersion, distilled water and green bean puree, by fifteen experienced $S$ s. The two psychophysical methods employed, detection thresholds and apparent taste intensity, showed good agreement. Salt sharply depressed apparent soumess in both media, as well as interfering with detection of sourness. Citric acid influenced apparent saltiness in a-complex manner, dependent upon concentration, media, method, and the individual $S$. In water, lower concentrations of acid generally enhanced and higher concentrations depressed sattiness. Within both media, half the $S$ s indicated on enhancement of saltiness with increasing acidity and half indicated the reverse. Higher acid levels interfered with detection of saltiness. This biphasic response to binary taste stimuli are discussed and compared with pertinent findings from previous titerature.

The conflicting conclusions in the literature relative to the interaction of the tastes of sweet, sour, salty. and bitter stimuli are due, In large part, to differences in the piychophysical methods and the range of stimulus concentrations employed. Data reported by Fabian and Blum (1943) surgented chemical specificity within a taste modality, e.s., the oweetness of fructose solutions was decreased by additions of lactic, malic, acetic, and tartaric acids but not by citrie and hydrochloric acids; the sweetness of sucrose solutions was enhanced by lactic, malic, citric, and tartarie acids but unaffected by aoetic and hydrochloric acids. Extensive testing in -our laboratory (Pangborn, 1965) showed that equisour concentrations of four organic acids depressed the sweetness of equisweet concentrations of four sugars. These data cast doubt upon the specificity of chemical configuration of stimull ellcitung a single taste quality, as the sour compounds depressed the tastes of sweet compounds independent of chemical structure.

Repeatedly, our data have shown a large between-S variability, both in direction and in magnitude of taste response (Pangborn 1961, 1962; Pangtorn \& Trabue, 1964; Pangborn Chrlsp, 1964). Marked inter-S differences in perception and in interpretation of complex taste stimull have been reported also by Gregson and McCowen (1963) and Gregson (1964), who points out that experiments which restrict themselves to measurement of threshold shifts cannot show whether intersensory effects are additive or multiplicative. A bimodal distribution of responses was obtained to mixtures of citric acid and sodium chloride, using lima bean purée as the test medium
(Pangborn \& Trabue, 1964). Five Ss found apparent saltiness to be decreased by increasing amounts of citric acid, whereas four Ss reported marked enhancement (Fig. 1). The study reported herein, therefore, was undertaken to determine whether this biphasic response to mixtures of salt and acid would be observed in other carriers. Furthermore, it was of interest to compare detection thresholds with judgments of apparent intensity of suprathreshold concentrations as influenced by the addition of a secondary compound.

\section{Subjects}

\section{METHOD}

Fifteen persons, nine male and six female departmentad employees, were selected on the basis of their ablitty to detect low concentrations of citric acid and of sodium chloride in aqueous solution.

\section{Stinuli}

The study was divided into four parts within each of the media of dispersion, distilled water and green bean purée:

Study 1: Effect of citric acid on apparent saltineas of sodium chloride;

Study 2: Effect of citric acid on detection of saltness of sodium chloride;

Study 3: Effect of sodium chloride on apparent sourness of citric acid;

Study 4: Effect of sodium chloride on detection of sourness of citric acld.

The concentrations were selected to range from barely perceptible to intense in aqueous solutions, with a corresponding selection made on the basis of stimulus intensity in the green bean purée medium. The exact levels of acid and salt used are indicated in the accompanying figures.

Aqueous solutions were prepared dally from freshly distilled water and stored in glass stoppered volumetric nasks. Reagent grades of citric acid and sodium chloride were used to prepare concentrated stock solutions from which measured volumes were taken for the final solutions. Samples were served in $50 \mathrm{ml}$ beakers at $22^{\circ} \mathrm{C}$, with each $\mathrm{S}$ recelving eight pairs per test session.

The green beans were of the Blue Lake variety, grown in Watsonville, Calif., and packed with no additives, by Stering Industry, Inc., Sacramento. The beans were packed in 9 oz (255 g) packages, quick frozen, and maintained at $-26^{\circ} \mathrm{C}$ until use. For each 


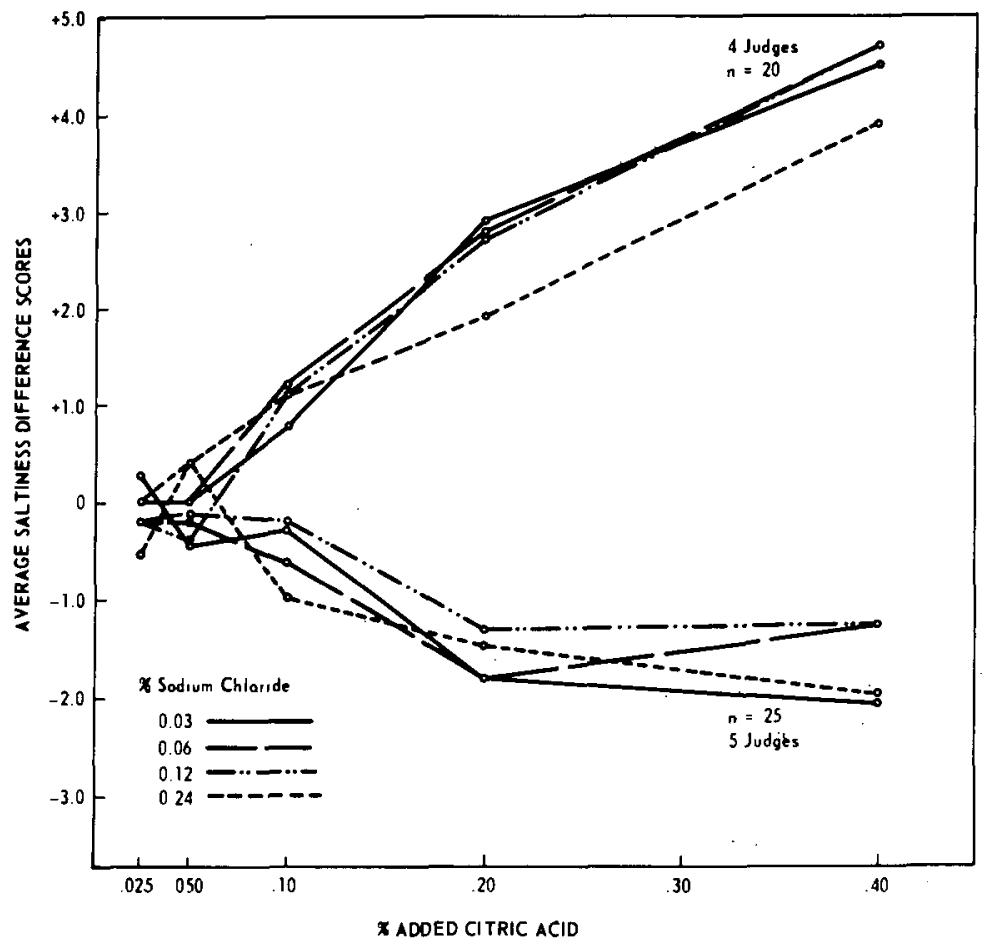

Fig. 1. Influence of citric acid on apparent saltiness in lima bean purée.

variable, $510 \mathrm{~g}$ of frozen beans were cooked in 800 $\mathrm{ml}$ of boiling distilled water for $13 \mathrm{~min}$. After draining for $3 \mathrm{~min}, 800 \mathrm{ml}$ of distilled water was added and the mixture was puréed in a Waring blender for $80 \mathrm{sec}$ at $110 \mathrm{~V}$. Weighed amounts of sodium chloride and measured volumes of a concentrated stock solution of citric acid were added to weighed portions of blended purée, then transferred to stainless steel saucepans. Stainless steel lids containing a thermometer were used to cover the pans, which were immersed in a water bath maintained at $80^{\circ} \mathrm{C}$ for a maximum of $45 \mathrm{~min}$ prior to serving. Samples of $35 \mathrm{~g}$ were served in coded, black lined porcelain cups placed in polystyrene blocks to maintain the temperature between 75 and $80^{\circ} \mathrm{C}$ during the test period. Each $S$ received four pairs of purée in randomized order at each session. Pureé and water solutions were served on alternate days throughout the study. Judging was performed under low red illumination to reduce visual differences. Ss were seated in individual partitioned booths maintained at $21 \pm 1^{\circ} \mathrm{C}$. Distilled water at $21^{\circ} \mathrm{C}$ was provided for oral rinsing and Ss were instructed not to swallow the sample or the rinse water. The composition of the samples was identified for the Ss at the termination of each session, and cake or cookies provided as "rewards." Test sessions were held between 2:00 and 3:00 PM Monday through Friday for a period of six months.

\section{Procedure}

A method of paired comparison, constant stimulus was used, wherein the composition of one member of a pair was constant throughout a series (Pangborn, 1961). For Studies 1 and 3, both samples within a pair contained the secondary taste compound, whereas only one contained the primary compound. Ss were asked to circle the code number of the sample within each pair that contained the greater apparent intensity of the secondary taste sensation. In addition, the degree of difference within a pair was indicated on a scale labeled slight, moderate, large, or extreme. These descriptive terms were converted to integers of $-1,-3,-5$, and -7 if the sample without the primary compound was selected and $+1,+3,+5$, and +7 if the sample containing the primary compound was selected as more intense. These difference scores were then submitted to analysis of variance.

In Studies 2 and 4, the Ss ability to detect the addition of the compound was determined in distilled water and in purée containing varying amounts of the secondary compound. Responses are expressed as percent correct identification.

\section{Study 1}

RESULTS

Citric acid influenced the apparent saltiness in a complex manner, as shown in Fig. 2. In water, lower concentrations of acid generally enhanced and higher concentrations depressed saltiness. With both media, the highest salt level demonstrated a marked decrease in intensity in the presence of higher acid levels. Inspection of the individual S's responses 
showed a distinct bimodality, of a much greater magnitude in green beans than in the aqueous solutions. Within both media, half the Ss indicated an enhancement of saltiness with increasing acidity and half indicated the reverse. A summary of the overall average for individual $S s$ is presented in Table 1. Since the studies were performed over a three year period, it was not possible, unfortunately, to retain the same Ss throughout. With the exception of S 8 , Ss 1 through 15 showed a consistency of direction and magnitude of response for the water and purée. The same holds for the water, green bean, and lima bean media for Ss $1,2,3,4,9$, and 10. A different pattern emerges for the tomato juice, where primarily positive responses, 1.e., enhancement of saltiness by acid, were obtained. The fact that tomato juice contains a higher natural acid content than the other media may have accounted for the different response pattern.
It is apparent from the data in Table 1 and the averaged values plotted in Figs. 1 and 2 that a much larger population of $\mathrm{Ss}$ should be tested to ascertain the validity of a true bimodality of response to these taste mixtures. It should be pointed out that binary mixtures of other taste modalities, e.g., sugar-salt, sugar-acid, did not result in differences in direction of response. Analysis of variance showed a large between-S variabllity, but this was due to magnitude, not direction, of response.

\section{Study 2}

Saltiness was easier to detect in nonacidified aqueous solutions than in those containing 0.02 or $0.04 \%$ citric acid (Fig. 3). In the purée, decidedly fewer correct responses were obtained in the presence of the highest concentration of acid, with a rather inconclusive relationship among the other three acid levels. In both media, it appears that the
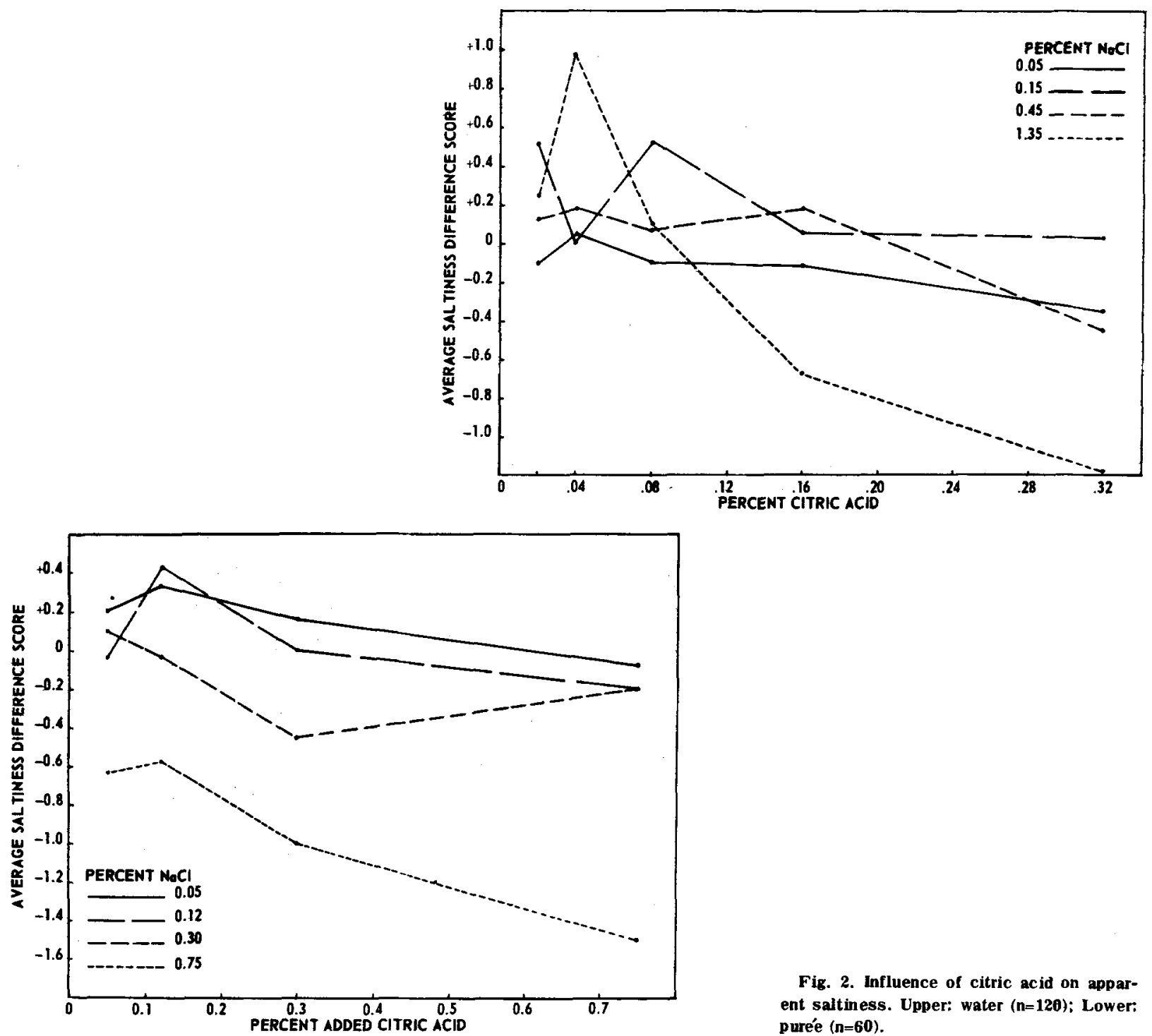

Fig. 2. Influence of citric acid on apparent saltiness. Upper: water $(n=12 \theta)$; Lower: purée $(n=60)$. 

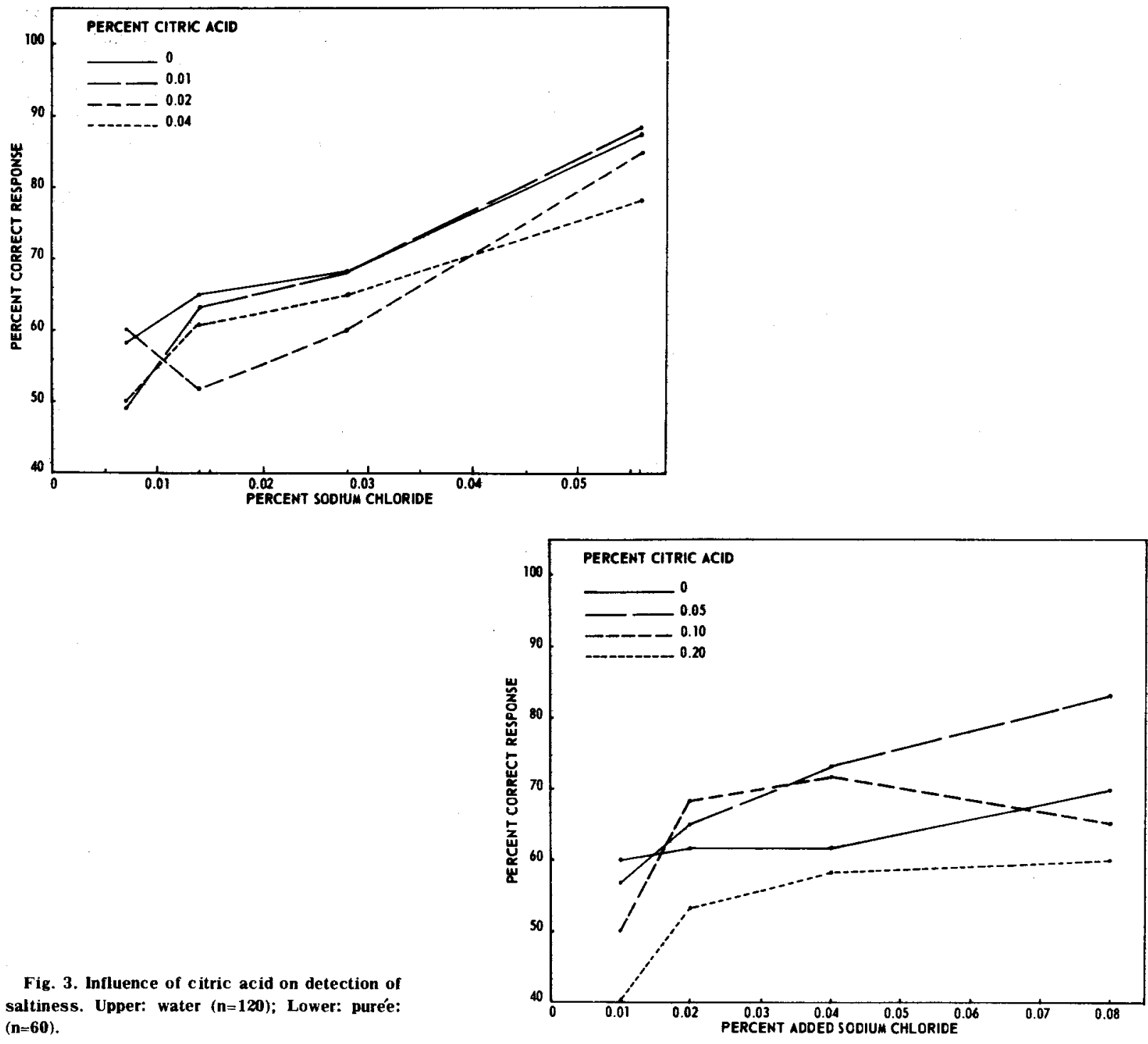

Fig. 3. Influence of citric acid on detection of saltiness. Upper: water $(n=120)$; Lower: purée: $(\mathbf{n}=\mathbf{6 0})$.

\section{Study 4}

highest acid level interfered with detection of the salty taste. Comparing Figs. 2 and 3 shows a general agreement between methods in that higher acid levels depressed both apparent saltiness and detection levels. The magnitude of this effect is greater in the puree; however, a different range of stimulus concentrations and different temperatures were used in the two media.

\section{Study 3}

Very clear-cut depression of apparent sourness could be attributed to increasing levels of sodium chloride with both media. Figure 4 shows that the highest levels of acid were diminished the most, with the two lowest levels of acid demonstrating little change in puree and only an initial drop in water. Comparable magnitudes of response were evident in the two media.

In water, detection of citric acid was impeded by increasing concentrations of salt. Figure 5 shows a less than conclusive relationship for purée, although $0.45 \%$ added salt resulted in fewer correct responses in three out of four levels of acid as compared with the unsalted series.

\section{General Results}

Comparison of the percentage correct responses for individual Ss with their respective apparent intensity scores did not give a significant correlation $(r=0.22)$. Relative to order of presentation within a pair in the detection studies, testing the control followed by the sapid solution (OX) did not differ significantly from the reverse tasting order (XO) $\left(X^{2}=0.679\right.$ for the aqueous medium and 0.375 for 


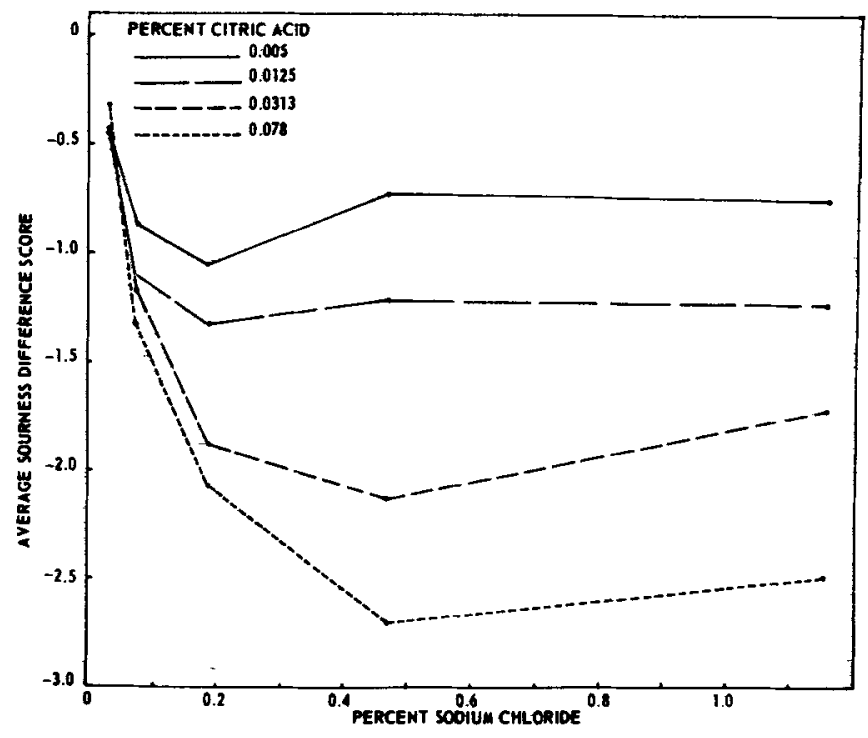

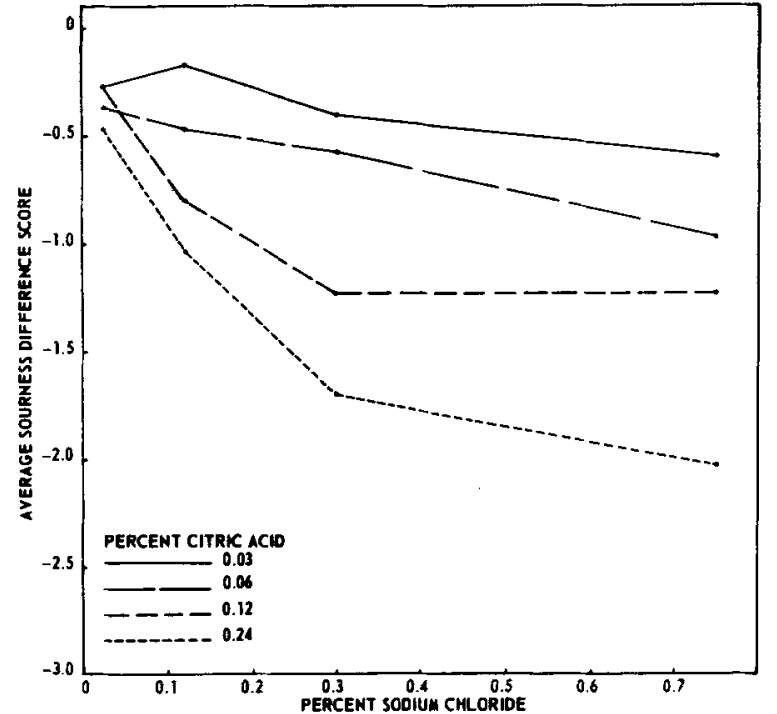

purée). The order of presentation of pairs within a test session had no influence on responses to samples of purée, but significantly affected responses in the aqueous medium. In Study 2, effect of acid on detection of saltiness, higher percent correct response was obtained for Pairs 1 and 2 than for Pairs 3 through $8\left(x^{2}=4.037, p<.05\right)$. Conversely, in Study 4, effect of salt on detection of sourness, higher percent correct response was obtained for Pairs 6,7 , and 8 than for the first five $\left(\chi^{2}=13.164\right.$, $p<.001)$.

\section{DISCUSSION}

Analyses of variance of the apparent intensity data (Studies 1 and 3) gave significant F-ratios for Ss, acid levels, and salt levels, but not for replications, indicating reproducibility of judgment among individual Ss. Most of the two- and three-way interactions involving replications were not significant. Table 2
Fig. 4. Influence of sodium chloride on apparent sourness. Upper: water $(n=120)$; Lower: purée $(n=60)$.

lists the F-ratios obtained from analysis of the detection data (Studies 2 and 4 ). Note that Ss always contributed high variability. Salt significantly affected detection of sourness in water but not in purée, whereas acid significantly affected detection of saltiness in purée but not in water. No logical explanation of this pattern is immediately apparent to us. It is evident, though, that extrapolation of results obtained from a simple aqueous medium to complex food media can be tenuous. It is encouraging, from the standpoint of the interactive effects of the two taste stimuli, that the two psychophysical methods employed herein gave comparable results. This increases one's confidence in the mechanism of the dual stimulation and in the consistency of response of the Ss. Unresolved, as yet, is the observed bimodality of response illustrated in Table 1 . It is not believed to be a mere artifact of the test method, as it was not manifested in salt-sugar or acid-sugar mixtures. 

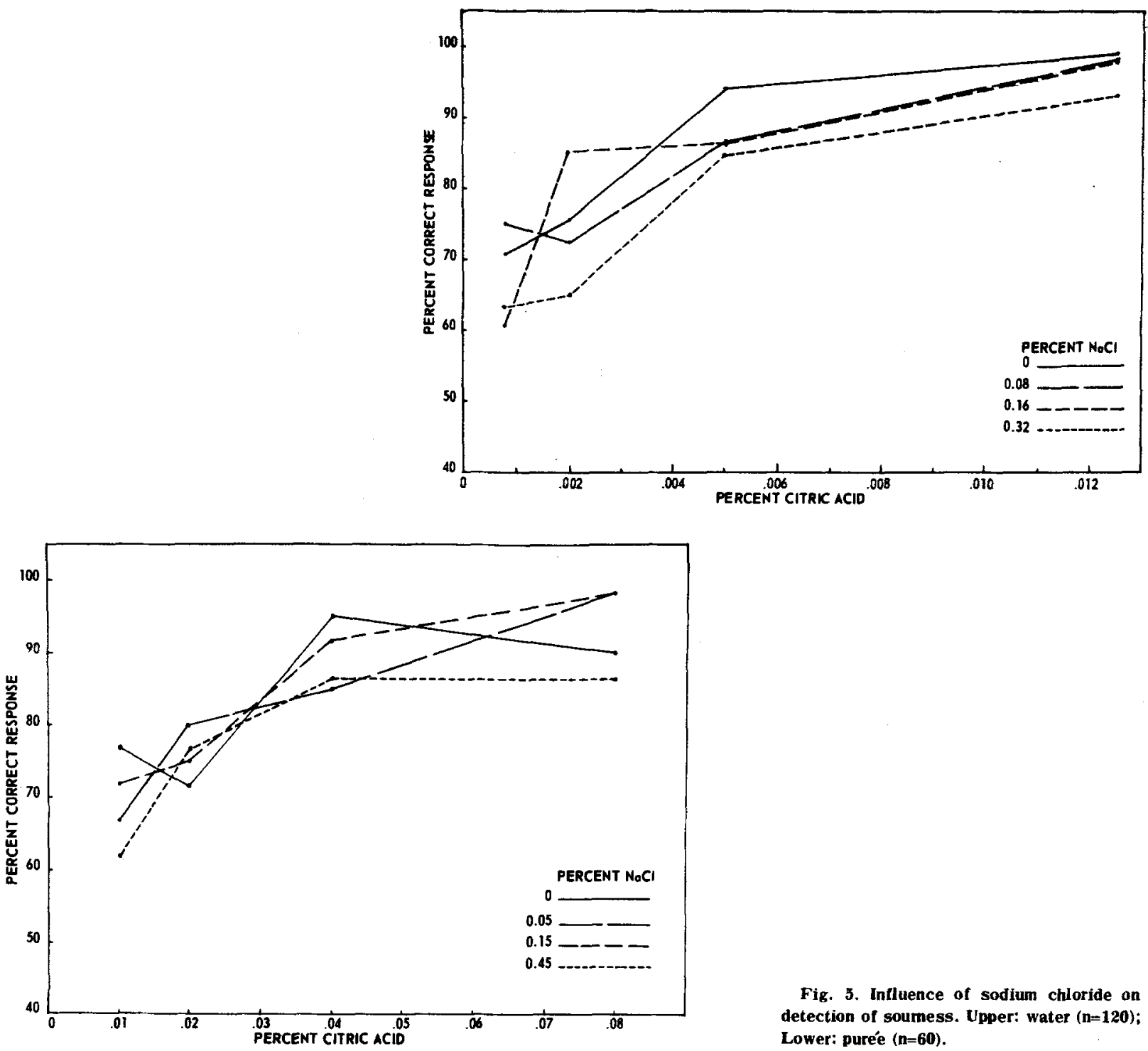

Fig. 5. Influence of sodium chloride an detection of soumess. Upper: water $(n=120)$; Lower: purée $(\mathbf{n}=60)$.

One should not discount the possibility of semantic misusage, i.e., confusion in assigning terms of "salty" and "sour" to sensations elicited by salts and acids.

The conflicting conclusions on taste properties of mixtures of salt and acid reported in earlier literature are not easily reconciled owing to the limited number of $\mathrm{Ss}$ tested and the varying types of psychophysical methods employed. For example, Kiesow (1894) used point stimuli of different sapid solutions on each side of the tongue and observed that salt and sour solutions exhibited simultaneous contrast of each for the other. The salt contrast effect on sour was greater than the sour contrast effect on salt.

Cragg (1937) reported that the sourness of a 0.005 $\mathrm{N}$ solution of hydrochloric acid was unchanged by the addition of $0.2 \mathrm{~N}$ sodium chloride, but an artifact could have resulted from combining two chloride ions. (This could have been avoided simply by the use of citric or acetic acid). The work of Fabian and Blum (1943) defies generalization, as they found that hydrochloric acid had no effect on sodium chloride, yet this sait was enhanced $20-25 \%$ by lactic, citric, malic, tartaric, and acetic acids. Addition of sodium chloride decreased citric, acetic, and hydrochloric acids by $10 \%$ and lactic, malic, and tartaric by $50 \%$. They gave no indication of between-S variability in direction of response.

Apparently Hahn and Ulbrich (1948) experienced some between-S variability in threshold shifts, as decreased thresholds for sodium chloride were obtained for one of three Ss in the presence of hydrochloric acid and for two of four Ss in the presence of acetic acid. All four Ss agreed that salt thresholds were lowered by picric acid. No changes in the thresholds of hydrochloric and acetic acids were observed upon addition of salt.

Anderson (1950) added threshold concentrations of 
Table 1. Effect of Citric Acid on Apparent Saltiness of Sodium Chloride in Four Media

(Values represent average intensity difference from a non-acidified control; negative values indicate depressing and positive values indicate enhancing effect of acid)

\begin{tabular}{lcccc}
$S$ & Water & $\begin{array}{c}\text { Green Bean } \\
\text { Purée }\end{array}$ & $\begin{array}{c}\text { Lima Bean } \\
\text { Puréé } a\end{array}$ & $\begin{array}{c}\text { Tomato } \\
\text { Juice } b\end{array}$ \\
\hline$n$ & 160 & 64 & 100 & 80 \\
1 & -0.4 & -1.8 & -1.1 & +1.5 \\
2 & -0.2 & -1.1 & -1.4 & +0.7 \\
3 & -0.8 & -1.2 & -0.2 & +0.8 \\
4 & -0.6 & -1.5 & -0.9 & -0.9 \\
5 & -2.9 & -3.4 & - & +2.0 \\
6 & -0.7 & -0.4 & - & +0.8 \\
7 & -0.1 & -0.3 & - & +0.6 \\
8 & +0.2 & -0.4 & - & - \\
9 & +0.9 & +0.9 & +0.9 & - \\
10 & +0.7 & +0.7 & +1.1 & - \\
11 & +1.8 & +2.7 & - & - \\
12 & +1.1 & +1.0 & - & - \\
13 & +0.5 & +0.9 & - & - \\
14 & +0.2 & +0.7 & - & - \\
15 & +0.3 & +0.2 & - & - \\
16 & - & - & - & +1.3 \\
17 & - & - & - & +0.8 \\
18 & - & - & - & +0.8 \\
19 & - & - & - & +0.3 \\
20 & - & - & - & -0.4 \\
21 & - & - & - & -0.3 \\
22 & - & - & +2.3 & - \\
23 & - & - & +2.1 & - \\
24 & - & - & -0.5 & - \\
\hline & - & - & &
\end{tabular}

a Pangborn and Trabue (1964).

b Pangborn and Chrisp (1964).

one component to varying concentrations of a second compound and found that thresholds for sodium chloride were raised for both his $\mathrm{Ss}$ in the presence of tartaric acid. However, the threshold for acid was slightly decreased by salt for one $S$ but raised in the presence of salt for the other $S$ (divergent directional responses with only two Ss!)

Using single sample presentation of suprathreshold concentrations of compounds representing the four basic tastes, Kamen et al (1961) conceded that results from the interaction of sodium chloride with citric acid were more complex than all the others. They

Table 2. Analysis of Variance of Number of Correct Responses to Mixtures of Sodium Chloride and Citric Acid. Detection Studies.

\begin{tabular}{|c|c|c|c|c|c|}
\hline \multirow{2}{*}{$\begin{array}{l}\text { Source } \\
\text { of } \\
\text { Voriation }\end{array}$} & \multirow[b]{2}{*}{ D.F. } & \multicolumn{2}{|c|}{ Study 2. Saltiness } & \multicolumn{2}{|c|}{ Study 4. Sourness } \\
\hline & & Water & Purée & Water & Purée \\
\hline Total & 239 & \multicolumn{4}{|c|}{ F-Ratios } \\
\hline Ss & 14 & $9.11^{* * *}$ & $2.43^{* *}$ & $12.58^{* * *}$ & $2.69 * *$ \\
\hline $\mathrm{NaCl}(\mathrm{N})$ & 3 & $42.34 * * *$ & $6.21 * * *$ & $4.81 * *$ & 1.19 \\
\hline Acid (A) & 3 & 2.05 & $4.93 * *$ & $62.08 * * *$ & $19.81 * * *$ \\
\hline Ss $\times N$ & 42 & $1.52^{*}$ & 1.01 & 1.11 & 0.62 \\
\hline Ss $\times A$ & 42 & 1.05 & 1.15 & $2.27 * * *$ & 1.07 \\
\hline$N \times A$ & 9 & 1.40 & 0.70 & $2.40 *$ & 1.00 \\
\hline Remainder & 126 & & & & \\
\hline
\end{tabular}

$*{ }^{*},{ }^{* *}$ Respectively significant at $p<.05, .01$, and .001 . concluded that sait had no monotonic effect upon sourness, but citric acid increased saltiness. They observed significant Salt by Acid interactions and Salt by Session interactions; however, since their 960 required replications were drawn from $700 \mathrm{Ss}$, it was not possible to analyze for between-S variability.

Of parallel interest are the findings of Feller et al (1965) on the flow rates of parotid salivary glands to mixtures of taste stimuli. They found that flow rates obtained from mixtures of citric acid and sodium citrate were less than the sum of the individual components, apparently due to the buffering effect causing a decreased hydrogen ion concentration. While the relation between salivary flow rates and perceived sourness has been reported (Chauncey et al, 1963), the corresponding relationship for salts has not been established.

\section{References}

Anderson, R. J. Taste thresholds in stimulus mixtures. Ph.D. thesis, University of Michigan, 1950.

Chauncey, H. H., Feller, R. P., \& Shannon, I. L. Effect of acid solutions on human gustatory chemoreceptors as determined by parotid gland secretion rate. Proc. Soc. exp. Biol. Med., 1963 , $112,917-923$

Cragg. L. H. The sour taste: Threshold values and accuracy, the effects of saltiness and sweetness. Trans. roy. Soc. Canada III, $1937,31,131-140$.

Fabian, F. W., \& Blum, H. B. Relative taste potency of some basic food constituents and their competitive and compensatory action. Food Res., 1943, 8, 179-193.

Feller, R. P., Sharon, I. M., Chauncey, H. H., \& Shannon, 1. L. Gustatory perception of sour, sweet, and salt mixtures using parotid gland flow rate. J. appl. Physiol., 1965, 20, 1314-1344.

Gregson, R. A. M. Modification of perceived relative intensities of acid tastes by ambient illumination changes. Aust. J. Psychol., 1964, 16, 190-199.

Gregson, R. A. M., \& McCowen, P. J. The relative perception of weak sucrose-citric acid mixtures. J. Food Sci, 1963, 28, 371. 378.

Hahn, H., \& Ulbrich, L. Eine systematische Untersuchung der Geschmacksschwellen. Pflüger's Arch. ges, Physiol., 1948, 250, 357-384.

Kamen, J. M., Pilgrim, F. J., Gutman, N. J., \& Kroll, B. J. Interactions of suprathreshold taste stimuli. J. exp. Psychol., 1961 , 4. 348-356.

Kiesow, F. Über die Wirkung des Cocain und der Gymnemasâure auf die Schleimhaut der Zunge und des Mundraums. Philosoph. Studien., 1894, 9, 510-527.

Pangbom, R. M. Taste interrelationships. II. Suprathreshold solutions of sucrose and citric acid. J. Food Sci., 1961, 26, 648-655.

Pangborm, R. M. Taste interrelationships. III. Suprathreshold solutions of sucrose and sodium chloride. J. Food Sci., 1962, $27,495-500$.

Pangbom, R. M., \& Trabue, I. M. Taste interrelationships. V. Sucrose, sodium chloride, and citric acid in Lima bean purée. $J$. Food Sci., 1964, 29, 233-240.

Pangbom, R. M., \& Chrisp, R. B. Taste interrelationships. VI. Sucrose, sodium chloride, and citric acid in canned tomato juice. J. Food Sci., 1964, 29, 490-498.

Pangbom, R. M. Taste interrelationships of organic acids and selected sugars. Proc. 1st int. Cong. Food Sci. \& Technol., London, Vol. III. J. M. Leitch (Ed.), New York: Gordon and Breach Science Publishers, 1965. Pp. 291-305.

(Accepted for publication July 3, 1967.) 